Vol 11, Issue 10, 2018

\title{
SYNTHESIS OF SOME NEW HETEROCYCLIC COMPOUNDS DERIVED FROM N-(Ñ-PHENYL GLYCYL) SACCHARIN AND STUDY THEIR BIOLOGICAL ACTIVITY
}

\author{
ESRAA M. ALI ${ }^{1 *}$, AHMED W. NASER ${ }^{1}$, MUTHANNA S. FARHAN ${ }^{2}$ \\ ${ }^{1}$ Department of Chemistry, College of Science, University of Baghdad, Baghdad, Iraq. ${ }^{2}$ Department of Pharmaceutical Chemistry, College of \\ Pharmacy, University of Baghdad, Baghdad, Iraq. Email: Chemistangle21@gmail.com
}

Received: 11 August 2018, Revised and Accepted: 11 September 2018

\section{ABSTRACT}

Objective: In the present work, a variety of new heterocyclic compounds namely aza- $\beta$ - lactam, cyclicimides, 1,3-thiazole, and 1,2,4-triazole was prepared.

Methods: Procedure includes the synthesis of aza- $\beta$ - lactam, cyclic imides, 1,3-thiazole, and 1,2,4-triazole. The synthesis was carried out in eleven steps using $\mathrm{N}$-(Ñ-substituted phenylglycyl) saccharin derivatives $(1 \mathrm{a}, \mathrm{b})$ as a starting material and converted to benzoic acid derivatives (2a,b) and then to ester derivatives $(3 a, b)$, which finally convers to benzohydrazide derivatives $(4 a, b)$. The cyclization of $(4 a, b)$ with carbon disulfide and hydrazine hydrate $(80 \%)$ in the presence of potassium hydroxide gives 1,2,4-triazole compounds $(5 \mathrm{a}, \mathrm{b})$, and subsequently $(5 \mathrm{a}, \mathrm{b})$ derivatives reacted with different aromatic aldehydes in the presence of few drops of glacial acetic acid to give Schiff bases (6a-f). Compounds (7a-b) was prepared by the reaction of compounds $(4 \mathrm{a}, \mathrm{b})$ with chloroacetyl chloride. 1,3-thiazole derivatives $(8 \mathrm{a}, \mathrm{b})$ were synthesized through the cyclization of compounds $(7 \mathrm{a}, \mathrm{b})$ with thiourea. Schiff bases (9a-f) were obtained by condensation of $(4 \mathrm{a}, \mathrm{b})$ with different aromatic aldehydes in the presence of few drops of glacial acetic acid. Aza- $\beta$-lactam compounds (10a-f) were prepared by the cycloaddition of Schiff-bases (9a-f) with phenyl isocyanate through [2+2] cycloaddition reaction. Reaction $(4 \mathrm{a}, \mathrm{b})$ with various acid anhydrides in presence of acetic acid gave the corresponding cyclic imide (11a-f).

Results: The results showed that compounds (5a) and (10e) have a good activity against Gram-positive bacterium and no activity against Gramnegative bacterium, compared to standard drugs (ciprofloxacin and amoxicillin), while compounds (8a) and (6b) have a high activity against fungi, compared to standard drugs (metronidazole benzoate), and the other tested compounds have low-to-moderate activity.

Conclusion: 1,2,4-triazole is a most potent assemblage of Gram-positive bacterium retardants and cyclic imides are a most potent assemblage of fungi retardants.

Keywords: 1,2,4-Triazole, Aza- $\beta$-lactam, Cyclic imide, Antibacterial, Antifungal.

(c) 2018 The Authors. Published by Innovare Academic Sciences Pvt Ltd. This is an open access article under the CC BY license (http://creativecommons. org/licenses/by/4. 0/) DOI: http://dx.doi.org/10.22159/ajpcr.2018.v11i10.29408

\section{INTRODUCTION}

The heterocyclic compounds are enjoying their importance as being the center of activity. The nitrogen-containing heterocyclic compounds were found in abundance in most of the medicinal compounds and the presence of three nitrogen heteroatoms in five-membered ring systems defines an interesting class of compounds [1]. The first Schiff base compounds were reported by Hugo Schiff in 1864. In recent years, the chemistry of Schiff bases contains $\mathrm{N}$-donor atom which has been extensively studied and has acquired a great interest because of the azomethine $\mathrm{C}=\mathrm{N}$ linkage essential for biological activity [2]. 1,2,4-triazoles constitute broad realization due to their useful application in different areas of biological activity, and as industrial intermediates, it is effectively used in polymers, dyestuff, photographic chemicals, and agricultural chemicals [3]. There is some biological activity of 1,2,4-triazole such as antimicrobial [4], anti-inflammatory [5], and antioxidant [6]. The $\beta$-lactam core is the fundament building block of an exceptionally large class of antibiotics that all share a common mode of action but have quite distinct properties in terms of spectrum pharmacokinetics and, to some extent, activity against resistant strains [7]. Cyclic imides have an important in the creation of novel medical materials, most widely method to synthesize cyclic imides by heating dicarboxylic acids or anhydrides with an amine and harsh thermal reaction conditions, and activation reagents are mandatory $[8,9]$. Thiazole and its derivatives comprise an important class of heterocyclic compounds with high biological activity such as antimicrobial, antipyretic, antiparasitic, antihistaminic, and antiviral activities [10].

\section{METHODS}

All chemicals were purchased from Fluka, BDH, and Merk. Melting points were recorded using electrothermal melting point apparatus. Fourier-transform infrared (FTIR) spectral data were recorded on a Shimadzu FTIR-8400 S spectrophotometer in the Department of Chemistry, College of Science, University of Baghdad. Nuclear magnetic resonance $\left({ }^{1} \mathrm{H}\right.$-NMR) spectrum was recorded in the Central Laboratory of Isfahan University and Sharif University of Technology, $400 \mathrm{MHz}$, using deuterated dimethyl sulfoxide (DMSO) as a solvent.

Step 1: Synthesis of $\mathrm{N}$-( $\tilde{\mathrm{N}}$-substituted phenylglycyl) saccharin derivatives $(1 \mathrm{a}, \mathrm{b})[11]$

A mixture of $\mathrm{N}$-(2-chloroacetyl) saccharin ( $0.01 \mathrm{~mole}, 2.6 \mathrm{~g})$, appropriate aromatic amine, namely $p$-toluidine and $o$-toluidine $(0.01 \mathrm{~mole})$, in $15 \mathrm{~mL}$ chloroform was refluxed for $6 \mathrm{~h}$. The formed precipitate was filtered, washed with water, dried, and finally recrystallized from chloroform to give compounds $(1 \mathrm{a}, \mathrm{b})$ respectively.

\section{$N$-[N-(p-toluylglycyl)] saccharin (1a)}

M.p $=220-223^{\circ} \mathrm{C}$, yield $=92 \%, \mathrm{R}_{\mathrm{r}}=0.673, \mathrm{IR}(\mathrm{KBr}) \mathrm{cm}^{-1}: 3093(\mathrm{~N}-\mathrm{H}), 2975$ (C-H alph), 1722 (C=0), 1296 (C-N amine).

\section{$N$-[Ñ-(o-toluylglycyl)] saccharin (1b)}

M.p $=218-221^{\circ} \mathrm{C}$, yield $=86 \%, \mathrm{R}_{\mathrm{f}}=0.630, \mathrm{IR}(\mathrm{KBr}) \mathrm{cm}^{-1}: 3091(\mathrm{~N}-\mathrm{H}), 2972$ (C-H alph), 1722 (C=0), 1296 (C-N amine). 
Step 2: Synthesis of benzoic acid derivatives (2a,b) [12]

A mixture of compounds (1a,b) (0.01 mole) and $10 \% \mathrm{NaOH}(20 \mathrm{~mL})$ was heated in a steam bath for $30 \mathrm{~min}$, then cooled it at room temperature, and neutralized by hydrochloric acid $(\mathrm{HCl})$, and the white precipitate appearing was filtered and then recrystallized from absolute ethanol, to give compounds $(2 \mathrm{a}, \mathrm{b})$ respectively.

O-[N-(p-toluylglycyl) sulfamoyl] benzoic acid (2a)

M.p $=140-143^{\circ} \mathrm{C}$, yield $=94 \%, \mathrm{R}_{\mathrm{f}}=0.599$, IR $(\mathrm{KBr}) \mathrm{cm}^{-1}: 3352(\mathrm{OH}), 3252$ (N-H), 3041 (C-H Ar), 2912 (C-H alpha), 1710 (C=0), 1587 (C=C Ar), 1542 (C=0 amide I), 1249 (C-O acid).

o-[N-(o-toluylglycyl) sulfamoyl] benzoic acid $(2 \mathrm{~b})$

M.p $=138-139^{\circ} \mathrm{C}$, yield $=84 \%, \mathrm{R}_{\mathrm{f}}=0.512$, IR (KBr) cm${ }^{-1}: 3351(\mathrm{OH}), 3252$ (N-H), 3041 (C-H Ar), 2912 (C-H alpha), 1710 (C=0), 1587 (C=C Ar), 1542 (C=Oamide I), 1249 (C-O acid). ${ }^{1} \mathrm{H}-\mathrm{NMR}$ and ${ }^{13} \mathrm{C}-\mathrm{NMR}$ spectra $\delta$ (ppm): 1.5 (s, 3H, Ar-CH3), 3.3 (s, 2H, O=C-CH2-NH), 7.1-8.2 (m, 9H, $\mathrm{Ar}-\mathrm{H}, \mathrm{NHSO}_{2}$ ), 13.7 (s, 1H, COOH). The 13C-NMR spectra 40 (Ar-CH3), 130-140 (Ar), 142 ( $\mathrm{C}=0$ ) amide, 168 ( $\mathrm{C}=0)$ acid.

\section{Step 3: Synthesis of ester derivatives $(3 a, b)[13]$}

Mixture of compounds (2a,b) (0.001 mole) and (30 mL) absolute ethanol, in the presence of concentrated sulfuric acid $(0.5 \mathrm{~mL})$, was refluxed for $8 \mathrm{~h}$, and after cooling and naturalized by sodium bicarbonate, the white precipitate was appeared and crystallized from absolute ethanol, to give compounds (3a,b), respectively.

Ethyl-o-[N-(p-toluylglycyl) sulfamoyl] benzoate (3a)

M.p $=278-279^{\circ} \mathrm{C}$, yield $=89 \%, \mathrm{R}_{\mathrm{f}}=0.564, \mathrm{IR}(\mathrm{KBr}) \mathrm{cm}^{-1}: 3238(\mathrm{~N}-\mathrm{H}), 3040$ (C-H Ar), 2918 (C-H alpha), 1716 (C=0 ester), 1618 (C=Oamide I), 1066 (C-O ester)

\section{Ethyl-o-[N-(o-toluylglycyl) sulfamoyl] benzoate $(3 \mathrm{~b})$}

M.p $=274-276^{\circ} \mathrm{C}$, yield $=77 \%, \mathrm{R}_{\mathrm{f}}=0.533$, IR $(\mathrm{KBr}) \mathrm{cm}^{-1}: 3249(\mathrm{~N}-\mathrm{H}), 3022$ (C-H Ar), 2908 (C-H alpha), 1726 (C=0 ester), 1620 (C=Oamide I), 1068 (C-O ester). While ${ }^{1} \mathrm{H}-\mathrm{NMR}$ and ${ }^{13} \mathrm{C}-\mathrm{NMR}$ spectra $\delta(\mathrm{ppm}): 1.5$ (s, 3H, Ar$\mathrm{CH}_{3}$ ), $3.2\left(\mathrm{q}, 2 \mathrm{H}, \mathrm{O}-\mathrm{CH}_{2}\right), 3.6\left(\mathrm{~s}, 2 \mathrm{H}, \mathrm{O}=\mathrm{C}-\mathrm{CH}_{2}-\mathrm{NH}\right), 7.1-8.2(\mathrm{~m}, 9 \mathrm{H}, \mathrm{Ar}-\mathrm{H}$, $\left.\mathrm{NHSO}_{2}\right)$, and ${ }^{13} \mathrm{C}-\mathrm{NMR} 30\left(\mathrm{CH}_{2}-\mathrm{CH}_{3}\right), 40\left(\mathrm{CH}_{3}-\mathrm{Ar}\right), 134-126(\mathrm{C}-\mathrm{Ar}), 174$ $(\mathrm{C}=0)$ ester.

\section{Step 4: Synthesis of benzohydrazide derivatives (4a,b) [14]}

To the solution of compounds (3a,b) ( 0.001 mole) in absolute ethanol $(15 \mathrm{~mL})$, hydrazine hydrate $(0.001$ mole) was added, and the mixture was refluxed for $4 \mathrm{~h}$; after cooling, the formed white precipitate was filtered and recrystallized by absolute ethanol, to give compounds (4a,b), respectively.

\section{$O$-[N-(p-toluylglycyl) sulfamoyl] benzohydrazide (4a)}

M.p $=320-322^{\circ} \mathrm{C}$, yield $=83 \%, \mathrm{R}_{\mathrm{f}}=0.601$, IR $(\mathrm{KBr}) \mathrm{cm}^{-1}: 3402-3305$ ( $\mathrm{NH}-\mathrm{NH}_{2}$ ), 3055 (C-H Ar), 2962 (C-H alpha), 1631 (C=O amide I), 1587 ( $\mathrm{C}=$ Oamide II), 1498 ( $\mathrm{C}=\mathrm{C}$ Ar).

\section{O-[N-(o-toluylglycyl) sulfamoyl] benzohydrazide (4b)}

M.p $=316-317^{\circ} \mathrm{C}$, yield $=76 \%, \mathrm{R}_{\mathrm{f}}=0.455$, IR $(\mathrm{KBr}) \mathrm{cm}^{-1}: 3402-3305(\mathrm{NH}-$ $\mathrm{NH}_{2}$ ), 3076 (C-H Ar), 2900 (C-H alpha), 1701 (C=0 Amide I), 1542 (C=0 amide II), 1487 (C=C Ar)

Step 5: Synthesis of 1,2,4-triazole derivatives (5a,b) [15]

A mixture of compounds $(4 \mathrm{a}, \mathrm{b})(0.001 \mathrm{~mole})$, potassium hydroxide ( 0.01 mole, $0.8 \mathrm{~g})$ and carbon disulfide $(0.1 \mathrm{~mole})$ in absolute ethanol, was refluxed for $4 \mathrm{~h}$ in water bath, then excess carbon disulfide was removed, after that, added ( 0.01 mole, $0.5 \mathrm{~g}$ ) hydrazine hydrate $80 \%$, and refluxed for $3 \mathrm{~h}$, after cooling, acidification with $20 \% \mathrm{HCl}$, and appearance precipitate was filtered and recrystallized from ethanol to give triazole derivatives $(5 a, b)$, respectively.
3-[o-(N-p-toluylglycyl)sulfamoyl]-4-amino-5-mercapto-1,2,4triazole (5a)

M.p $=152-154^{\circ} \mathrm{C}$, yield $=91 \%, \mathrm{R}_{\mathrm{f}}=0.576$, IR $(\mathrm{KBr}) \mathrm{cm}^{-1}: 3213,3267(\mathrm{NH}-$ $\mathrm{NH}_{2}$ ), 3087 (C-H Ar), 2929 (C-H alpha), 2661 (SH), 1645 (C=Oamide I), $1596(\mathrm{C}=\mathrm{N}), 1502\left(\mathrm{C}=\mathrm{C}\right.$ Ar). The ${ }^{1} \mathrm{H}-\mathrm{NMR}$ spectrum $\delta(\mathrm{ppm}): 1.6(\mathrm{~s}, 3 \mathrm{H}$, Ar- $\left.\mathrm{CH}_{3}\right), 3.6\left(\mathrm{~s}, 2 \mathrm{H}, \mathrm{NH}_{2}\right), 4.2\left(\mathrm{~s}, 2 \mathrm{H}, \mathrm{CH}_{2}-\mathrm{C}=0\right), 5.2(\mathrm{~s}, 1 \mathrm{H}, \mathrm{CH}$-triazole ring), 7.1-8.1 (m, 9H, Ar-H, $\left.\mathrm{NHSO}_{2}\right), 12.7(\mathrm{~s}, 1 \mathrm{H}, \mathrm{SH})$.

3-[o-(N-o-toluylglycyl)sulfamoyl]-4-amino-5-mercapto-1,2,4triazole $(5 b)$

M.p $=144-146^{\circ} \mathrm{C}$, yield $=86 \%, \mathrm{R}_{\mathrm{f}}=0.539$, IR $(\mathrm{KBr}) \mathrm{cm}^{-1}: 3213,3267(\mathrm{NH}-$ $\mathrm{NH}_{2}$ ), 3087 (C-H Ar), 2929 (C-H alpha), 2661 (SH), 1643 (C=Oamide I), $1596(\mathrm{C}=\mathrm{N}), 1502(\mathrm{C}=\mathrm{C}$ Ar)

\section{Step 6: Synthesis of Schiff bases (6a-f) derived from compounds} $(5 a, b)[16]$

A mixture of compounds (5a,b) (0.001 mole), appropriatearomaticaldehyde, namely 4,4-dimethylaminobenzaldehyde, 4-methoxybenzaldehyde, and 2,4-dimethoxybenzaldehyde, and a few drops of glacial acetic acid in $10 \mathrm{~mL}$ absolute ethanol, was refluxed for $5 \mathrm{~h}$. The formed precipitate after cooling was filtered, dried, and recrystallized from ethanol to give compounds (6a-f).

3-[o-(N-p-toluylglycyl)sulfamoyl]-4-(4,4-dimethylaminobenz ylidine)-5-mercapto-1,2,4-triazole (6a)

M.p $=177-175^{\circ} \mathrm{C}$, yield $=90 \%, \mathrm{R}_{\mathrm{f}}=0.423$, IR (KBr) cm${ }^{-1}: 3253(\mathrm{NH}), 3023$ (C-H Ar), 2918 (C-H alpha), 1602 (C=N imine), 1525(C=N).

3-[o-(N-p-toluylglycyl)sulfamoyl]-4-(4-methoxybenzylidine)-5mercapto-1,2,4-triazole (6b)

M.p $=185-183^{\circ} \mathrm{C}$, yield $=87 \%, \mathrm{R}_{\mathrm{f}}=0.451$, IR $(\mathrm{KBr}) \mathrm{cm}^{-1}: 3256(\mathrm{NH}), 3022$ (C-H Ar), 2935 (C-H alpha), 1602 (C=N imine), 1537 (C=N).

3-[o-(N-p-toluylglycyl)sulfamoyl]-4-(2,4-dimethoxybenzylidine)5-mercapto-1,2,4-triazole (6c)

M.p $=166-165^{\circ} \mathrm{C}$, yield $=81 \%, \mathrm{R}_{\mathrm{f}}=0.498, \mathrm{IR}(\mathrm{KBr}) \mathrm{cm}^{-1}: 3218(\mathrm{NH}), 3006$ (C-H Ar), 2925(C-H alpha), 1604 (C=N imine), 1573(C=N).

3-[o-(N-o-toluylglycyl)sulfamoyl]-4-(4,4-dimethylaminobenz ylidine)-5-mercapto-1,2,4-triazole (6d)

M.p $=173-171^{\circ} \mathrm{C}$, Yield $=86 \%, \mathrm{R}_{\mathrm{r}}=0.383$, IR $(\mathrm{KBr}) \mathrm{cm}^{-1}: 3200(\mathrm{NH}), 3023$ (C-H Ar), 2920 (C-H alpha), 1604 (C=N imine), $1523(\mathrm{C}=\mathrm{N})$

3-[o-(N-o-toluylglycyl)sulfamoyl]-4-(4-methoxybenzylidine)-5mercapto-1,2,4-triazole (6e)

$\mathrm{M} . \mathrm{p}=179-178^{\circ} \mathrm{C}$, yield $=81 \%, \mathrm{R}_{\mathrm{r}}=0.376, \quad \mathrm{IR}(\mathrm{KBr}) \mathrm{cm}^{-1}: 3255(\mathrm{NH})$, 3020(C-H Ar), 2923 (C-H alpha), 1614 (C=N imine), 1589 (C=N).

3-[o-(N-o-toluylglycyl)sulfamoyl]-4-(2,4-dimethoxybenzylidine)5-mercapto-1,2,4-triazole (6f)

M.p $=156-153^{\circ} \mathrm{C}$, yield $=82 \%, \mathrm{R}_{\mathrm{f}}=0.364, \mathrm{IR}(\mathrm{KBr}) \mathrm{cm}^{-1}: 3200(\mathrm{NH}), 3001$ (C-H Ar), 2920 (C-H alpha), 1612 (C=N imine), 1502 (C=N)

Step 7: Synthesis of N-chloroacetyl benzohydrazide derivatives (7a,b) [17]

A mixture of compounds (4a,b) (0.001 mole) dissolved in $10 \mathrm{~mL}$ of dry benzene was stirred in ice bath for $30 \mathrm{~min}$, then chloroacetyl chloride was added by dropwise then stirring at room temperature for $1 / 2 \mathrm{~h}$, and the produced white precipitate was filtrated and recrystallized from benzene and methanol $(3: 2)$ to give compounds $(7 \mathrm{a}, \mathrm{b})$ respectively.

O-[N-(p-toluylglycyl)sulfamoyl]- $\tilde{N}$-(chloroacetyl) benzohydrazide (7a)

M.p $=147-145^{\circ} \mathrm{C}$, yield $=90 \%, \mathrm{R}_{\mathrm{f}}=0.341, \mathrm{IR}(\mathrm{KBr}) \mathrm{cm}^{-1}: 3184(\mathrm{NH}), 3043$ (C-H Ar), 2958(C-H alpha), 1703, 1610 (C=0), 794 (C-Cl). 
O-[N-(o-toluylglycyl)sulfamoyl]-Ñ-(chloroacetyl)benzohydrazide (7b)

M.p $=132-130^{\circ} \mathrm{C}$, Yield =83\%, R $=0.387$, IR $(\mathrm{KBr}) \mathrm{cm}^{-1}: 3182(\mathrm{NH}), 3043$ (C-H Ar), 2854 (C-H alpha), 1724, 1612 (C=0), 794 (C-Cl).

\section{Step 8: Synthesis of 1,3-thiazole derivatives (8a,b)}

A mixture of compound (7a,b) (0.001 mole) and 0.005 mole, $0.8 \mathrm{~g}$, of thiourea, in $10 \mathrm{~mL}$ absolute ethanol, was refluxed for $4 \mathrm{~h}$, and after cooling at room temperature, produced precipitate was filtered and recrystallized from ethanol to give compounds $(8 \mathrm{a}, \mathrm{b})$ respectively.

O-[N-(p-toluylglycyl)sulfamoyl]-Ñ-(2-amino-1,3-thiazole-5-yl) benzohydrazide (8a)

M.p $=192-194^{\circ} \mathrm{C}$, yield $=79 \%, \mathrm{R}_{\mathrm{f}}=0.749$, IR $(\mathrm{KBr}) \mathrm{cm}^{-1}: 3390-3200$ ( $\mathrm{NH}-\mathrm{NH}_{2}$ ), 3060 (C-H Ar), 2800 (C-H alpha), 1714 (C=0 amide I), 1514 $\left(\mathrm{C}=\mathrm{C}\right.$ Ar), $1602(\mathrm{C}=\mathrm{N}), 1514\left(\mathrm{C}=\mathrm{O}\right.$ amide II). The ${ }^{1} \mathrm{H}-\mathrm{NMR}$ spectrum of compound (8a) showed the following characteristic signals $\delta(\mathrm{ppm})$ : 1.6 (s, $\left.\left.3 \mathrm{H}, \mathrm{CH}_{3}-\mathrm{Ar}\right), 3.3\left(\mathrm{~s}, 2 \mathrm{H}, \mathrm{O}=\mathrm{C}-\mathrm{CH}_{2}-\mathrm{NH}\right), 3.7\right)\left(\mathrm{s}, 2 \mathrm{H}, \mathrm{NH}_{2}\right), 4.1(\mathrm{~s}$, $1 \mathrm{H}, \mathrm{CH}$ thiazole ring), 7.1-7.9 (m, 9H, Ar-H, $\mathrm{NHSO}_{2}$ ), 11.7 (s, $1 \mathrm{H}, \mathrm{O}=\mathrm{C}-$ NH-NH).

O-[N-(o-toluylglycyl)sulfamoyl]-Ñ-(2-amino-1,3-thiazole-5-yl) benzohydrazide $(8 b)$

M.p $=186-185^{\circ} \mathrm{C}$, yield $=71 \%, \mathrm{R}_{\mathrm{f}}=0.518, \mathrm{IR}(\mathrm{KBr}) \mathrm{cm}^{-1}: 3390-3251(\mathrm{NH}-$ $\mathrm{NH}_{2}$ ), 3047 (C-H Ar), 2962 (C-H alpha), 1710 (C=O amide I), 1429 (C=C Ar), $1606(\mathrm{C}=\mathrm{N}), 1518(\mathrm{C}=\mathrm{O}$ amide $\mathrm{II})$.

Step 9: Synthesis of Schiff bases (9a-f) derived from compounds (4a,b) [18]

The following Schiff-bases (9a-f) were prepared under similar conditions as for compounds (6a-f).

$O$-[N-(p-toluylglycyl)sulfamoyl]-Ñ-(4,4-dimethylaminobenz ylidine) benzamide (9a)

M.p $=60-61^{\circ} \mathrm{C}$, yield $=88 \%, \mathrm{R}_{\mathrm{f}}=0.345, \mathrm{IR}(\mathrm{KBr}) \mathrm{cm}^{-1}: 3277(\mathrm{NH}), 3100$ (C-H Ar), 2918 (C-H alpha), 1602 (C=O amide I), 1523 (C=N), 1517 (C=O amide II).

$O$-[N-(p-toluylglycyl)sulfamoyl]-Ñ-(4-methoxybenzylidine) benzamide (9b)

M.p $=79-81^{\circ} \mathrm{C}$, yield $=84 \%, \mathrm{R}_{\mathrm{f}}=0.467, \mathrm{IR}(\mathrm{KBr}) \mathrm{cm}^{-1}: 3246(\mathrm{NH}), 3040$ (C-H Ar), 2942 (C-H alpha), 1623 (C=O amide I), 1602 (C=N), 1556 (C=O amide II).

O-[N-(p-toluylglycyl)sulfamoyl]-Ñ-(2,4-dimethoxybenzylidine) benzamide (9c)

M.p $=86-88^{\circ} \mathrm{C}$, yield $=89 \%, \mathrm{R}_{\mathrm{f}}=0.391, \mathrm{IR}(\mathrm{KBr}) \mathrm{cm}^{-1}: 3286(\mathrm{NH}), 3006$ (C-H Ar), 2975 (C-H alpha), 1604 (C=O amide I), 1579 (C=N), 1502 (C=0 amide II). The ${ }^{1} \mathrm{H}-\mathrm{NMR}$ spectrum $\delta$ (ppm): 1.5 (s, 3H, $\left.\mathrm{Ar}^{-\mathrm{CH}_{3}}\right), 3.4(\mathrm{~s}, 3 \mathrm{H}$, $\left.\mathrm{OCH}_{3}\right), 3.8\left(\mathrm{~s}, 2 \mathrm{H}, \mathrm{O}=\mathrm{C}_{-}-\mathrm{CH}_{2}-\mathrm{NH}\right), 6.6(\mathrm{~s}, 1 \mathrm{H}, \mathrm{CH}=\mathrm{N}), 7.1-7.9(\mathrm{~m}, 9 \mathrm{H}, \mathrm{Ar}-\mathrm{H}$, $\left.\mathrm{NHSO}_{2}\right), 8.8(\mathrm{~s}, 1 \mathrm{H}, \mathrm{O}=\mathrm{C}-\mathrm{NH}-\mathrm{N})$.

O-[N-(o-toluylglycyl)sulfamoyl]-Ñ-(4,4-dimethylaminobenz ylidine) benzamide (9d)

M.p $=62-64^{\circ} \mathrm{C}$, yield $=83 \%, \mathrm{R}_{\mathrm{f}}=0.624$, IR $(\mathrm{KBr}) \mathrm{cm}^{-1}: 3174(\mathrm{NH}), 3074$ (C-H Ar), 2979 (C-H alpha), 1639 (C=O amide I), 1602 (C=N), 1523 (C=O amide II). The ${ }^{1} \mathrm{H}-\mathrm{NMR}$ spectrum $\delta$ (ppm): $1.6\left(\mathrm{~s}, 3 \mathrm{H}, \mathrm{Ar}-\mathrm{CH}_{3}\right), 3.3(\mathrm{~s}, 6 \mathrm{H}$, $\left.\left(\mathrm{CH}_{3}\right)_{2} \mathrm{~N}\right), 3.8\left(\mathrm{~s}, 2 \mathrm{H}, \mathrm{O}=\mathrm{C}-\mathrm{CH}_{2}-\mathrm{NH}\right), 6.7(\mathrm{~s}, 1 \mathrm{H}, \mathrm{CH}=\mathrm{N}), 7.1-7.9(\mathrm{~m}, 9 \mathrm{H}, \mathrm{Ar}-$ $\left.\mathrm{H}, \mathrm{NHSO}_{2}\right), 8.4(\mathrm{~s}, 1 \mathrm{H}, \mathrm{O}=\mathrm{C}-\mathrm{NH}-\mathrm{N})$.

O-[N-(o-toluyl glycyl) sulfamoyl]- $\tilde{N}$-(4-methoxy benzylidine) benzamide $(9 e)$

M.p $=71-74^{\circ} \mathrm{C}$, yield $=82 \%, \mathrm{R}_{\mathrm{f}}=0.581$, IR (KBr) cm${ }^{-1}: 3145(\mathrm{NH}), 3008$ (C-H Ar), 2972 (C-H alpha), 1631 (C=O amide I), 1600 (C=N), 1510 ( $\mathrm{C}=\mathrm{O}$ amide II).
O-[N-(o-toluylglycyl)sulfamoyl]-Ñ-(2,4-dimethoxybenzylidine) benzamide ( $9 f$ )

M.p $=90-92^{\circ} \mathrm{C}$, yield $=86 \%, \mathrm{R}_{\mathrm{f}}=0.468$, IR (KBr) cm${ }^{-1}: 3290(\mathrm{NH}), 3008$ (C-H Ar), 2844 (C-H alpha), 1600 (C=0 amide I), 1596 (C=N), 1502 ( $\mathrm{C}=0$ amide II).

\section{Step 10: Synthesis of Aza- $\beta$-lactam derivatives (10a-f) [19]}

A mixture of Schiff bases (9a-f) (0.001 mole) and phenyl isocyanate $(0.001$ mole, $0.05 \mathrm{~g})$ in chloroform $(15 \mathrm{~mL})$ was refluxed for $6 \mathrm{~h}$. The solvent was removed and the residue was treated with a mixture of 1:1 ethyl acetate and petroleum ether. The resultant precipitate was filtered and dried to give compounds (10a-f), respectively.

$O-[N-(p-t o l u y l g l y c y l)$ sulfamoyl]- $N$-[2-oxo-3-phenyl-4-(4,4dimethylamino phenyl-1,3-diazatidine-1-yl)] benzamide (10a)

M.p $=102-104^{\circ} \mathrm{C}$, yield $=87 \%, \mathrm{R}_{\mathrm{f}}=0.321$, IR (KBr) cm $\mathrm{cm}^{-1}: 3307(\mathrm{NH}), 3060$ (C-H Ar), 2912 (C-H alpha), 1708 (C=0 Aza- $\beta$-lactam), 1668 (C=0 amide I), 1552 ( $\mathrm{C}=0$ amide II).

$O$-[N-(p-toluylglycyl)sulfamoyl]-N-[2-oxo-3-phenyl-4-(4-methoxy phenyl-1,3-diazatidine-1-yl)] benzamide (10b)

M.p $=114-115^{\circ} \mathrm{C}$, yield $=83 \%, \mathrm{R}_{\mathrm{f}}=0.433$, IR $(\mathrm{KBr}) \mathrm{cm}^{-1}: 3400(\mathrm{NH})$, 3050 (C-H Ar), 2991 (C-H alpha), 1704 (C=0 Aza- $\beta$-lactam), 1622 (C=0 amide I), 1560 ( $\mathrm{C}=0$ amide II).

$O$-[N-(p-toluylglycyl)sulfamoyl]-N-[2-oxo-3-phenyl-4-(2,4methoxyphenyl-1,3-diazatidine-1-yl)] benzamide (10c)

M.p $=123-125^{\circ} \mathrm{C}$, yield $=80 \%, \mathrm{R}_{\mathrm{f}}=0.544$, IR (KBr) cm${ }^{-1}: 3222(\mathrm{NH}), 3099$ (C-H Ar), 2921 (C-H alpha), 1701 (C=0 Aza- $\beta$-lactam), 1672 (C=0 amide I), 1541 ( $\mathrm{C}=0$ amide II). The ${ }^{1} \mathrm{H}$-NMR spectrum $\delta$ (ppm): 1.2 (s, $1 \mathrm{H}, \mathrm{NH}-\mathrm{Ar}), 1.6\left(\mathrm{~s}, 3 \mathrm{H}, \mathrm{CH}_{3}-\mathrm{Ar}\right), 3.6\left(\mathrm{~s}, 3 \mathrm{H}, p-\mathrm{OCH}_{3}\right), 3.8\left(\mathrm{~s}, 3 \mathrm{H}, o-\mathrm{OCH}_{3}\right)$, $4.1\left(\mathrm{~s}, 2 \mathrm{H}, \mathrm{O}=\mathrm{C}-\mathrm{CH}_{2}\right), 6.5(\mathrm{~s}, 1 \mathrm{H}, \mathrm{N}-\mathrm{CH}$-aza- $\beta$-lactam), 6.9-9.5 (m, 9H, $\mathrm{Ar}-\mathrm{H}, \mathrm{NHSO}_{2}$ ), 11.1 (s, 1H, N-NH-C=0).

O-[N-(o-toluylglycyl)sulfamoyl]- $N$-[2-oxo-3-phenyl-4-(4,4dimethylamino phenyl-1,3-diazatidine-1-yl)] benzamide (10d) M.p $=107-109^{\circ} \mathrm{C}$, yield $=80 \%, \mathrm{R}_{\mathrm{f}}=0.331$, IR $(\mathrm{KBr}) \mathrm{cm}^{-1}: 3288(\mathrm{NH})$, 3061 (C-H Ar), 2979 (C-H alpha), 1710 (C=0 Aza- $\beta$-lactam), 1672 (C=0 amide I), 1552 ( $\mathrm{C}=0$ amide II). The ${ }^{1} \mathrm{H}-\mathrm{NMR}$ spectrum $\delta(\mathrm{ppm}): 1.2$ (s, $1 \mathrm{H}, \mathrm{NH}-\mathrm{Ar}), 1.6\left(\mathrm{~s}, 3 \mathrm{H}, \mathrm{CH}_{3}-\mathrm{Ar}\right), 3.4\left(\mathrm{~s}, 6 \mathrm{H},\left(\mathrm{CH}_{3}\right)_{2}-\mathrm{N}\right), 4.1(\mathrm{~s}, 2 \mathrm{H}, \mathrm{O}=\mathrm{C}-$ $\left.\mathrm{CH}_{2}\right), 6.5$ (s, $1 \mathrm{H}, \mathrm{N}-\mathrm{CH}$-aza- $\beta$-lactam), 6.9-9.5 (m, 9H, Ar-H, $\mathrm{NHSO}_{2}$ ), 11.4 (s, $1 \mathrm{H}, \mathrm{N}-\mathrm{NH}-\mathrm{C}=0)$.

O-[N-(O-toluylglycyl)sulfamoyl]- $\mathrm{N}$-[2-oxo-3-phenyl-4-(4methoxyphenyl-1,3-diazatidine-1-yl)] benzamide (10e)

M.p $=118-120^{\circ} \mathrm{C}$, yield $=77 \%, \mathrm{R}_{\mathrm{f}}=0.554$, IR (KBr) cm${ }^{-1}: 3294(\mathrm{NH}), 3136$ (C-H Ar), 2900 (C-H alpha), 1708 (C=0 Aza- $\beta$-lactam), 1647 (C=0 amide I), 1556 ( $\mathrm{C}=\mathrm{O}$ amide II).

O-[N-(o-toluylglycyl)sulfamoyl]-N-[2-oxo-3-phenyl-4-(2,4methoxyphenyl-1,3-diazatidine-1-yl)] benzamide (10f)

M.p $=129-132^{\circ} \mathrm{C}$, yield $=72.7 \%, \mathrm{R}_{\mathrm{f}}=0.342$, IR $(\mathrm{KBr}) \mathrm{cm}^{-1}: 3290(\mathrm{NH})$, 3091 (C-H Ar), 2959 (C-H alpha), 1701 (C=0 Aza- $\beta$-lactam), 1645 (C=O amide I), 1502 ( $\mathrm{C}=0$ amide II).

Step 11: Synthesis of cyclicimide derivatives (11a-f) [20] A mixture of compounds (4a,b) (0.001 mole), appropriate acid anhydride, namely maleic anhydride, succinic anhydride, and phthalic anhydride ( $0.001 \mathrm{~mole})$, in $15 \mathrm{~mL}$ acetic acid, was refluxed for $24 \mathrm{~h}$. The formed precipitate was filtered, dried, and recrystallized from acetic acid to give compounds (11a-f), respectively.

11a-N-[o-[(Ñ - $p$-toluylglycyl) sulfamoyl] benzamedo succinimide: b.p $=121^{\circ} \mathrm{C}$, yield $=60 \%, \mathrm{R}_{\mathrm{f}}=0.686, \mathrm{IR}(\mathrm{KBr}) \mathrm{cm}^{-1}: 3207(\mathrm{NH}), 3051(\mathrm{C}-\mathrm{H}$ Ar), 2920 ( $\mathrm{C}-\mathrm{H}$ alpha), 1724 ( $\mathrm{C}=0$ cyclic imide), 1765 ( $\mathrm{C}=\mathrm{O}$ amide $\mathrm{I}$ ), 1602 ( $\mathrm{C}=\mathrm{C}$ Ar), 1525 ( $\mathrm{C}=0$ amide II). 
11b-N-[o-[(Ñ $-p$-toluylglycyl) sulfamoyl]benzamedomaleimide: $\mathrm{m} . \mathrm{p}=227-$ $228^{\circ} \mathrm{C}$, yield $=79 \%, \mathrm{R}_{\mathrm{f}}=0.574, \mathrm{IR}(\mathrm{KBr}) \mathrm{cm}^{-1}: 3257(\mathrm{NH}), 3068(\mathrm{C}-\mathrm{H} \mathrm{Ar})$, 2900 (C-H alpha), 1797 ( $\mathrm{C}=0$ cyclic imide), 1647 ( $\mathrm{C}=\mathrm{O}$ amide I), 1577 ( $\mathrm{C}=\mathrm{C} \mathrm{Ar}), 1552$ ( $\mathrm{C}=\mathrm{O}$ amide $\mathrm{II})$.

11c-N-[o-[(Ñ- $p$-toluylglycyl)sulfamoyl] benzamedophthalimide: $\mathrm{m} . \mathrm{p}=236-238^{\circ} \mathrm{C}$, yield $=83 \%, \mathrm{R}_{\mathrm{f}}=0.666$, IR $(\mathrm{KBr}) \mathrm{cm}^{-1}: 3271(\mathrm{NH})$, 3011 (C-H Ar), 2810 (C-H alpha), 1747 ( $\mathrm{C}=0$ cyclic imide), 1660 (C=0 amide I), 1600 ( $\mathrm{C}=\mathrm{C}$ Ar), 1556 ( $\mathrm{C}=0$ amide II). The ${ }^{1} \mathrm{H}-\mathrm{NMR}$ spectrum showed the following characteristic signals $\delta$ (ppm): Compound (11c): $1.6\left(\mathrm{~s}, 3 \mathrm{H}, \mathrm{Ar}-\mathrm{CH}_{3}\right), 3.2$ (s, 2H, Ar- $\left.\mathrm{NH}-\mathrm{CH}_{2}\right), 7.4-8.2(\mathrm{~m}, 13 \mathrm{H}, \mathrm{Ar}-\mathrm{H}$, $\mathrm{NHSO}_{2}$ ), 11.5 (s, 1H, C=O-NH-N-).

11d-N-[o-[( $\mathrm{N}-o$-toluylglycyl)sulfamoyl] benzamedo succinimide: m.p $=210-208^{\circ} \mathrm{C}$, yield $=76 \%, \mathrm{R}_{\mathrm{f}}=0.534$, IR (KBr) cm $\mathrm{cm}^{-1}: 3207(\mathrm{NH})$, 3011(C-H Ar), 2902 (C-H alpha), 1724 ( $\mathrm{C}=0$ cyclic imide), 1600 (C=0 amide I), 1577 ( $\mathrm{C}=\mathrm{C} \mathrm{Ar}$ ), $1561(\mathrm{C}=0$ amide II)
11e-N-[o-[( $\mathrm{N}$ - $o$-toluylglycyl)sulfamoyl] benzamedomaleimide: $\mathrm{m} . \mathrm{p}=243-$ $245^{\circ} \mathrm{C}$, yield $=79 \%, \mathrm{R}_{\mathrm{f}}=0.681$, IR (KBr) cm cm $^{-1} 3255(\mathrm{NH}), 3031$ (C-H Ar), 2950 (C-H alpha), 1703 ( $\mathrm{C}=0$ cyclic imide), 1670 ( $\mathrm{C}=0$ amide I), 1550 ( $\mathrm{C}=\mathrm{C} \mathrm{Ar}), 1523$ ( $\mathrm{C}=0$ amide II).

$11 \mathrm{f}-\mathrm{N}-[0-[(\tilde{N}-o$-toluylglycyl)sulfamoyl]benzamedophthalimide: $\mathrm{m} . \mathrm{p}=251-249^{\circ} \mathrm{C}$, yield $=80 \%, \mathrm{R}_{\mathrm{f}}=0.497$, IR $(\mathrm{KBr}) \mathrm{cm}^{-1}: 3274(\mathrm{NH})$, 3018 (C-H Ar), 2996 (C-H alpha), 1701 (C=0 cyclic imide), 1660 (C=0 amide I), 1567 ( $\mathrm{C}=\mathrm{C} \mathrm{Ar}$ ), 1556 ( $\mathrm{C}=\mathrm{O}$ amide II).

\section{BIOLOGICAL ACTIVITY}

Anti-bacterial and anti-fungal test [21]

The test was performed according to the disk diffusion assay. The synthesized compounds have been studied for their antimicrobial activity in vitro against four tested bacteria (Staphylococcus epidermidis and Staphylococcus aureus as Gram-positive bacteria and Klebsiella pneumonia and Escherichia coli as Gram-negative bacteria) and one type of fungi (Candida albicans).

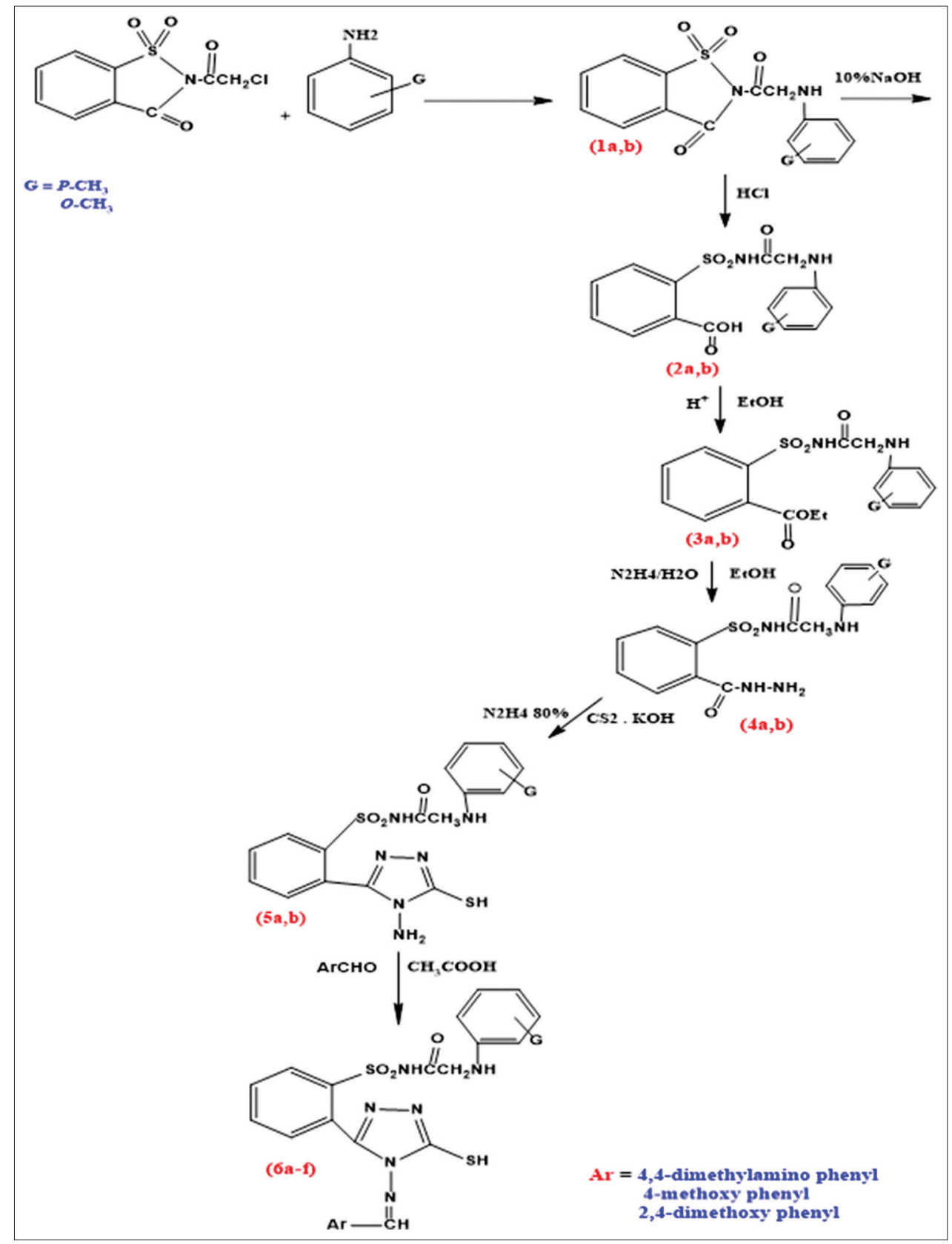

Scheme 1: Synthesis route for the preparation of compounds (5a-b) and (6a-f) 


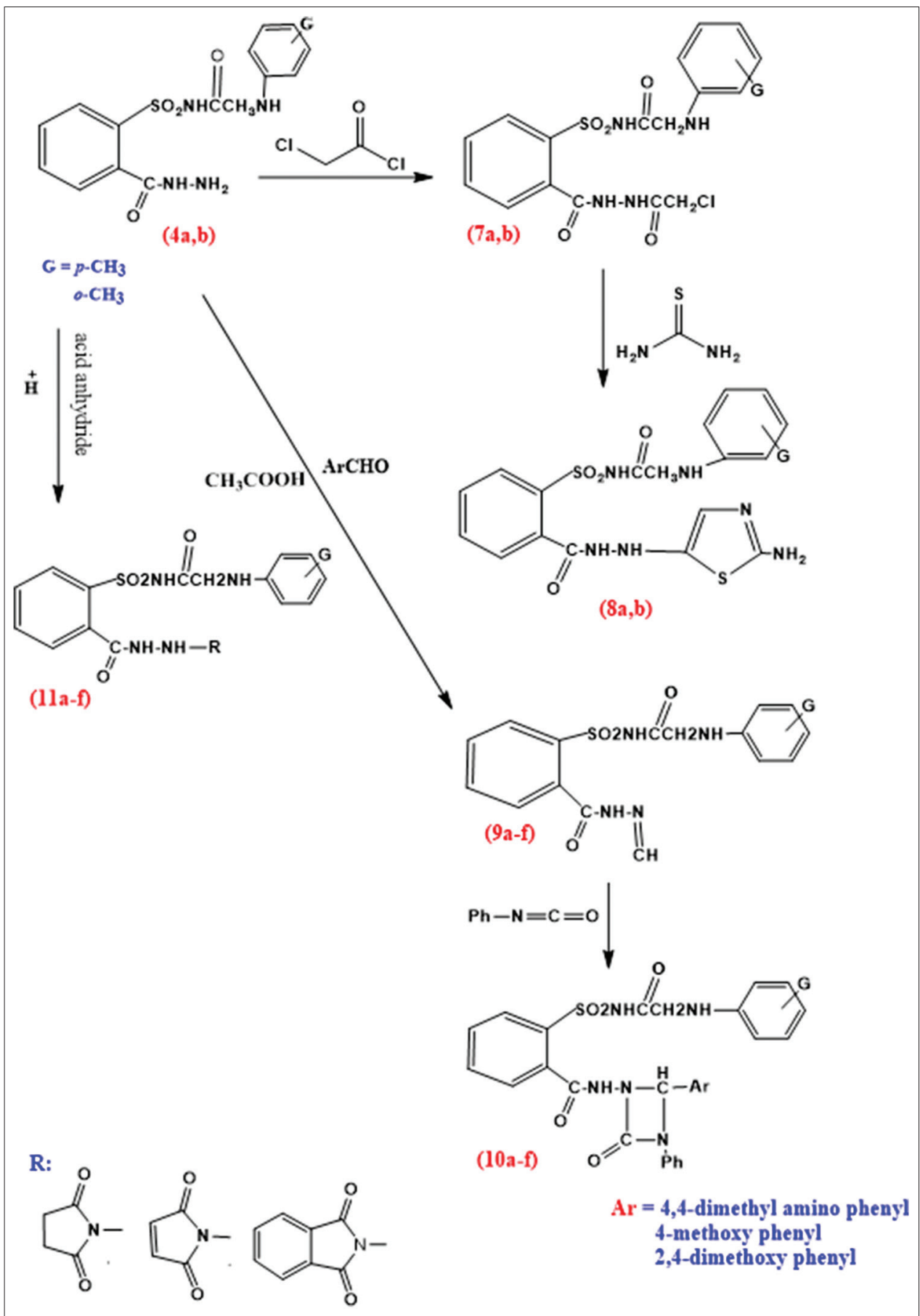

Scheme 2: Synthesis route for the preparation of compounds (8a-b), (10a-f), and (11a-f)

The agar and Petri dishes were sterilized by autoclaving for $15 \mathrm{~min}$ at $121^{\circ} \mathrm{C}$. The agar plates were surface inoculated uniformly from the broth culture of the tested microorganisms. The suitably spaced apart holes in a solidified medium were made all $6 \mathrm{~mm}$ in diameter, in which they were filled with $100 \mu \mathrm{L}$ of prepared compounds $(1 \mathrm{mg}$ of the compound dissolved in $1 \mathrm{~mL}$ of DMSO solvent), and ciprofloxacin was used as a standard. The incubation of these plates was for $24 \mathrm{~h}$ at $37^{\circ} \mathrm{C}$ (Scheme 1 and 2).

\section{RESULTS AND DISCUSSION}

$\mathrm{N}$-(Ñ-substituted phenylglycyl) saccharin derivatives $(1 \mathrm{a}, \mathrm{b})$ as a starting material were prepared according to the literature method [11]. It reacts with sodium hydroxide to give benzoic acid derivatives $(2 \mathrm{a}, \mathrm{b})$, benzoic acid derivatives react with absolute ethanol in the presence of concentrated $\mathrm{H}_{2} \mathrm{SO}_{4}$ to give ester derivatives $(3 \mathrm{a}, \mathrm{b})$, and then it reacts with $80 \%$ hydrazine hydrate to give benzohydrazide derivatives $(4 \mathrm{a}, \mathrm{b})$. The disappearance of $\left(\mathrm{C}=0\right.$ ester) stretching band at $1715-1726 \mathrm{~cm}^{-1}$ and the appearance of new stretching bands at 3402-3305 $\mathrm{cm}^{-1}$ and $1631 \mathrm{~cm}^{-1}$ which are due to $\left(\mathrm{NH}-\mathrm{NH}_{2}\right)$ and ( $\mathrm{C}=\mathrm{O}$ amide), respectively, are attributed to the formation of benzohydrazide derivative $(4 a, b)$. The cyclization of $(4 \mathrm{a}, \mathrm{b})$ with carbon disulfide in the presence of potassium hydroxide then added hydrazine hydrate to give the corresponding 1,2,4-triazole compound (5a,b). IR spectrum show the absence of the stretching band at (1631) $\mathrm{cm}-1$ for $\mathrm{C}=0$ amide and appearance of stretching band for $(\mathrm{C}=\mathrm{N})$ at $1596 \mathrm{~cm}-1$ it was good evidence for the formation of 1,2,4- triazole derivative, 1H-NMR spectrum of compound 
Table 1: Antibacterial activity of compounds (8b-12f)

\begin{tabular}{|c|c|c|c|c|}
\hline \multirow{2}{*}{$\begin{array}{l}\text { Compound } \\
\text { number }\end{array}$} & \multicolumn{4}{|c|}{ Zone of inhibition in $(\mathrm{mm})$, concentration $(1 \mathrm{mg} / \mathrm{mL})$} \\
\hline & $\begin{array}{l}\text { Gram-positive } \\
\text { Staphylococcus aureus }\end{array}$ & $\begin{array}{l}\text { Gram-positive } \\
\text { Staphylococcus epidermis }\end{array}$ & $\begin{array}{l}\text { Gram-negative } \\
\text { Escherichia coli }\end{array}$ & $\begin{array}{l}\text { Gram-negative } \\
\text { Klebsiella pneumonia }\end{array}$ \\
\hline $8 b$ & 10 & 9 & 8 & - \\
\hline $8 c$ & - & 10 & 9 & - \\
\hline $8 e$ & 16 & 10 & - & 8 \\
\hline $8 f$ & 9 & 10 & 10 & - \\
\hline $10 \mathrm{a}$ & 10 & 10 & 9 & - \\
\hline $11 \mathrm{a}$ & 9 & 20 & 8 & - \\
\hline $11 b$ & 10 & 9 & 8 & - \\
\hline $12 \mathrm{a}$ & 10 & 10 & 9 & 14 \\
\hline $12 b$ & 9 & 10 & 9 & - \\
\hline $12 \mathrm{~d}$ & - & 10 & 9 & - \\
\hline $12 \mathrm{f}$ & 9 & 9 & 8 & - \\
\hline Ciprofloxacin & 25 & 30 & 30 & 30 \\
\hline Amoxicillin & 18 & 15 & 17 & 20 \\
\hline
\end{tabular}

Table 2: Antifungal activity of compounds (7f-12b)

\begin{tabular}{ll}
\hline Compound no & $\begin{array}{l}\text { Zone of inhibition in }(\mathbf{m m}), \\
\text { concentration }(\mathbf{1} \mathbf{~ m} / \mathbf{m L}) \\
\text { Candida albicans }\end{array}$ \\
\hline $7 \mathrm{f}$ & 19 \\
$10 \mathrm{a}$ & 24 \\
$11 \mathrm{a}$ & 16 \\
$12 \mathrm{~b}$ & 18 \\
Metronidazole benzoate & 22 \\
\hline
\end{tabular}

(5a,b) shown the following characteristic signars $1.6\left(\mathrm{~s}, 3 \mathrm{H}, \mathrm{Ar}-\mathrm{CH}_{3}\right)$, $3.6\left(\mathrm{~s}, 2 \mathrm{H}, \mathrm{NH}_{2}\right), 4.2$ (s, $\left.2 \mathrm{H}, \mathrm{NH}_{2}-\mathrm{CH}_{2}-\mathrm{C}=0\right), 5.2$ (s, $1 \mathrm{H}, \mathrm{CH}$-triazole ring), 7.1-8.1 (m, 9H, Ar-H, NHSO $\left.{ }_{2}\right), 12.7$ (s, 1H, SH). Schiff base derivatives (6a-f) from 1,2,4-triazole are prepared by reacting compounds $(5 \mathrm{a}, \mathrm{b})$ with some aromatic aldehyde such as 4,4-dimethylaminobenzaldehyde, 4-methoxybenzaldehyde, and 2,4-dimethoxy benzaldehyde in the presence of few drops of glacial acetic acid. IR spectrum of compounds (6a-f) shows the disappearance of $\left(\mathrm{NH}_{2}\right)$ stretching band at 3402-3305 $\mathrm{cm}^{-1}$ and appearance bands of $(\mathrm{C}=\mathrm{N})$ at $1602-1624 \mathrm{~cm}^{-1}$ and $(\mathrm{N}-\mathrm{H})$ at $3253 \mathrm{~cm}^{-1}$. N-(chloroacetyl) benzohydrazide derivatives $(7 \mathrm{a}, \mathrm{b})$ were prepared by the reaction of compounds $(4 \mathrm{a}, \mathrm{b})$ with chloroacetyl chloride; IR spectrum of compounds $(7 \mathrm{a}, \mathrm{b})$ shown disappearance of (NH-NH${ }_{2}$ ) overlapping with (NH) $3402-3305 \mathrm{~cm}^{-1}$ and appearance of $1703-1610 \mathrm{~cm}^{-1}(\mathrm{C}=0)$ and $794 \mathrm{~cm}^{-1}(\mathrm{C}-\mathrm{Cl}) .1,3$-thiazole compounds $(8 \mathrm{a}, \mathrm{b})$ were produced by the reaction of compounds $(7 \mathrm{a}, \mathrm{b})$ with thiourea. IR spectrum of compounds $(8 \mathrm{a}, \mathrm{b})$ shown the disappearance of stretching bands 1702-1610 $\mathrm{cm}^{-1}(\mathrm{C}=0)$ and $794 \mathrm{~cm}^{-1}(\mathrm{C}-\mathrm{Cl})$, and appearance of 1606-1602 $\mathrm{cm}^{-1}(\mathrm{C}=\mathrm{N})$ and $3900-3200 \mathrm{~cm}^{-1}$ (N-H-NH ${ }_{2}$; ${ }^{1} \mathrm{H}-\mathrm{NMR}$ spectrum of compounds $(8 \mathrm{a}, \mathrm{b})$ shown: 1.6 (s, $\left.3 \mathrm{H}, \mathrm{CH}_{3}-\mathrm{Ar}\right), 3.3\left(\mathrm{~s}, 2 \mathrm{H}, \mathrm{O}=\mathrm{C}-\mathrm{CH}_{2}-\mathrm{NH}\right), 3.7\left(\mathrm{~s}, 2 \mathrm{H}, \mathrm{NH}_{2}\right), 4.1(\mathrm{~s}, 1 \mathrm{H}, \mathrm{CH}$ thiazolering), 7.1-7.9 (m, 9H, Ar- $\mathrm{H}, \mathrm{NHSO}_{2}$ ), and $11.7(\mathrm{~s}, 1 \mathrm{H}, \mathrm{O}=\mathrm{C}-$ NH-NH). Schiff bases (9a-f) were obtained by condensation of $(4 a, b)$ with 4,4-dimethylaminobenzaldehyde, 4-methoxybenzaldehyde, and 2,4-dimethoxy benzaldehyde in the presence of few drops of glacial acetic acid. IR spectrum data of compounds (9a-f) shown disappearance of $\left(\mathrm{NH}-\mathrm{NH}_{2}\right)$ overlapping with $(\mathrm{NH}) 3402-3305 \mathrm{~cm}^{-1}$ and appearance of $(\mathrm{C}=\mathrm{N}) 1602-1523 \mathrm{~cm}^{-11},(\mathrm{C}=0) 1623-1600 \mathrm{~cm}^{-1}$, the $1 \mathrm{H}-\mathrm{NMR}$ spectrum showed the following characteristic signals $\delta(\mathrm{ppm})$ : Compound (9c): $1.5\left(\mathrm{~s}, 3 \mathrm{H}, \mathrm{Ar}-\mathrm{CH}_{3}\right), 3.4\left(\mathrm{~s}, 3 \mathrm{H}, \mathrm{OCH}_{3}\right), 3.8(\mathrm{~s}, 2 \mathrm{H}, \mathrm{O}=\mathrm{C}-$ $\left.\mathrm{CH}_{2}-\mathrm{NH}\right), 6.6(\mathrm{~s}, 1 \mathrm{H}, \mathrm{CH}=\mathrm{N}), 7.1-7.9\left(\mathrm{~m}, 9 \mathrm{H}, \mathrm{Ar}-\mathrm{H}, \mathrm{NHSO}_{2}\right), 8.8(\mathrm{~s}, 1 \mathrm{H}$, $\mathrm{O}=\mathrm{C}-\mathrm{NH}-\mathrm{N})$, compound (9d): $1.6\left(\mathrm{~s}, 3 \mathrm{H}, \mathrm{Ar}-\mathrm{CH}_{3}\right), 3.3\left(\mathrm{~s}, 6 \mathrm{H},\left(\mathrm{CH}_{3}\right)_{2} \mathrm{~N}\right), 3.8$ (s, 2H, O=C- $\left.-\mathrm{CH}_{2}-\mathrm{NH}\right), 6.7(\mathrm{~s}, 1 \mathrm{H}, \mathrm{CH}=\mathrm{N}), 7.1-7.9\left(\mathrm{~m}, 9 \mathrm{H}, \mathrm{Ar}-\mathrm{H}, \mathrm{NHSO}_{2}\right)$, $8.4(\mathrm{~s}, 1 \mathrm{H}, \mathrm{O}=\mathrm{C}-\mathrm{NH}-\mathrm{N})$. Aza- $\beta$-lactam compounds (10a-f) were prepared by the cycloaddition of Schiff bases (9a-f) with phenyl isocyanate via. $(2+2)$ cycloaddition reaction, IR spectrum data shown disappearance of $(\mathrm{C}=\mathrm{N}) 1602-1523 \mathrm{~cm}^{-1},(\mathrm{C}=0) 1623-1600 \mathrm{~cm}^{-1}$ and appearance of $1710-1701 \mathrm{~cm}^{-1}$ (C=0 aza- $\beta$-lactam), ${ }^{1} \mathrm{H}$-NMR spectrum of compound (10c): 1.2 (s, 1H, NH-Ar), 1.6 (s, 3H, $\mathrm{CH}_{3}-\mathrm{Ar}$ ), 3.6 (s, 3H, p-OCH $), 3.8$ (s, $\left.3 \mathrm{H}, o-\mathrm{OCH}_{3}\right), 4.1\left(\mathrm{~s}, 2 \mathrm{H}, \mathrm{O}=\mathrm{C}-\mathrm{CH}_{2}\right), 6.5(\mathrm{~s}, 1 \mathrm{H}, \mathrm{N}-\mathrm{CH}$-aza- $\beta$-lactam), 6.99.5 (m, 9H, Ar-H, NHSO ${ }_{2}$ ), 11.1 (s, $\left.1 \mathrm{H}, \mathrm{N}-\mathrm{NH}-\mathrm{C}=0\right)$. Compound (10d): $1.2(\mathrm{~s}, 1 \mathrm{H}, \mathrm{NH}-\mathrm{Ar}), 1.6\left(\mathrm{~s}, 3 \mathrm{H}, \mathrm{CH}_{3}-\mathrm{Ar}\right), 3.4\left(\mathrm{~s}, 6 \mathrm{H},\left(\mathrm{CH}_{3}\right)_{2}-\mathrm{N}\right), 4.1(\mathrm{~s}, 2 \mathrm{H}$, $\left.\mathrm{O}=\mathrm{C}-\mathrm{CH}_{2}\right), 6.5\left(\mathrm{~s}, 1 \mathrm{H}, \mathrm{N}-\mathrm{CH}\right.$-aza- $\beta$-lactam), 6.9-9.5 (m, 9H, Ar-H, $\mathrm{NHSO}_{2}$ ), $11.4(\mathrm{~s}, 1 \mathrm{H}, \mathrm{N}-\mathrm{NH}-\mathrm{C}=\mathrm{O})$. Reaction (4a-b) with various acid anhydrides, namely succinic anhydride, maleic anhydride, and phthalic anhydride, in the presence of acetic acid gave the corresponding cyclicimide (11af), IR spectrum of compounds (11a-f) shown disappearance of (NH$\mathrm{NH} 2$ ) overlapping with $(\mathrm{NH})$ at $3402-3305 \mathrm{~cm}^{-1}$ and appearance of 1797-1724 $\mathrm{cm}^{-1}(\mathrm{C}=0)$ cyclicimide. ${ }^{1} \mathrm{H}-\mathrm{NMR}$ spectrum of compound (11c) shown: $1.6\left(\mathrm{~s}, 3 \mathrm{H}, \mathrm{Ar}-\mathrm{CH}_{3}\right), 3.2\left(\mathrm{~s}, 2 \mathrm{H}, \mathrm{Ar}-\mathrm{NH}-\mathrm{CH}_{2}\right), 7.4-8.2(\mathrm{~m}$, $13 \mathrm{H}, \mathrm{Ar}-\mathrm{H}, \mathrm{NHSO}_{2}$ ), and 11.5 (s, $1 \mathrm{H}, \mathrm{C}=0-\mathrm{NH}-\mathrm{N}-$ ). The antimicrobial activity done on four different strains of bacteria and one type of fungi. The results showed that compounds (11a) and (8e) have a good activity against Gram-positive bacterium and no activity against Gram-negative bacterium, compared to standards drugs (ciprofloxacin, amoxicillin), while compounds (10a) and (12b) have a high activity against fungi, compared to standard drugs (metronidazole benzoate), and the other tested compounds have low to moderate activity.

\section{Antibacterial and antifungal assay}

Some of the prepared compounds were tested against two strain of Gram-positive bacteria such as S. aureus and S. epidermidis, two strains of Gram-negative bacteria such as K. pneumonia and E. coli, and one strain of fungi $C$. albicans. The agar and Petri dishes were sterilized by autoclaving for $15 \mathrm{~min}$ at $121^{\circ} \mathrm{C}$. The agar plates were surface inoculated uniformly from the broth culture of the tested microorganisms. The suitably spaced apart holes in the solidified medium were made all $6 \mathrm{~mm}$ in diameter, in which were filled with $100 \mu \mathrm{l}$ of prepared compounds ( $1 \mathrm{mg}$ of the compound dissolved in $1 \mathrm{~mL}$ of DMSO solvent), ciprofloxacin, amoxicillin was used as the standard for bacterial test and metroniolazorbenzoate stander for fungi. The incubation of these plates was for $24 \mathrm{~h}$ at $37^{\circ} \mathrm{C}$ (Table 1 ana 2) [21].

\section{CONCLUSION}

5 a is a most potent assemblage of Gram-positive bacterium retardants and $8 \mathrm{a}$ is a most potent assemblage of fungi retardants.

\section{AUTHORS' CONTRIBUTIONS}

Ahmed W. Naser suggested the idea, starting material, and thesis of work. Muthanna S. Farhan suggested the type of biological activity. Esraa M. Ali suggested step 6 and step 7 of work, prepared compounds, write, and review the article.

\section{CONFLICTS OF INTEREST}

The authors declare that they have no conflicts of interest. 


\section{REFERENCES}

1. Kumaraswamy B, Ranjith KT, Narasimha S, Vasudeva RN. Synthesis, characterization and in vitro microbial evaluation of regioisomers of allyl phenyl ethers derived 1, 2, 4-Triazoles, Int J Pharm Pharm Sci 2014;6:572-5.

2. Jithendra KK, Krishnamurthy GS, Sunil K. Synthesis, characterization, in vitro antimicrobial, anthelmintic and docking studies of new 2-[(E)$\{[4-(1 \mathrm{H}-1,2,4$-Triazole-1ylmethyl)phenyl]imino $\}$ methyl]phenol, and their complexes with 3D metal ions. Int J Pharm Pharm Sci 2016;8:134-9.

3. El-Badih AG, Hassan MM, Elsayed AA, Bahgat RM. Synthesis and antibacterial activity of some new 4-aniline-5-phenyl-4H-1,2,4triazole-3-thiol derivatives. Arabian J Chem 2016;9:S1654-9.

4. Ibtisam KJ, Wissam Kh, Salwa A, Abdulla HM. Synthesis and characterization of some new of thiazolidine, 1,2,4-triazole, 1,3,4-thiadiazole, semicarbazide, oxazoline and a study of their biological activity. Kerbala J pharm Sci 2012;3:213-22.

5. Mazin NM, Shaker AN. Evaluation of the anti-inflammatory activity and ulcerogenic lability of 5-(3-Chloro-1-benzathine-2-yl)-4-Phenyl4H-1,2,4-triazole-3-thiol. Bas J Vet Res 2012;11:122-7.

6. Stefania FB, Diana CI, Gabriel S, Valentina U, Valentina U, Constantin D, Valeria R. Synthesis and antioxidant activity evaluation of new compounds from hydrazinecarbothioamide and 1,2,4-triazole class containing diarylsulfone and 2,4-difluorophenyl moieties. Int J Mol Sci 2014; 15:10908-25.

7. David SH. Physical Biochemistry: Principles and applications. Chem Eur J 18(9):1250-56.

8. Ahlam MA, Suroor AB. Synthesis, characterization and biological activity study of N-substituted sulfonamido maleimides substituted with different heterocycles. Baghdad Sci J 2010;7:641-53.

9. Min W, Jianmin L, Jiping MA, Zhe Z, Feng W. Cuprous oxide catalyzed oxidative $\mathrm{C}-\mathrm{C}$ bond cleavage for $\mathrm{C}-\mathrm{N}$ bond formation: Synthesis of cyclic imides from ketones and amines. Chem Int 2015;54:14061-5.
10. Mohsen A, Ali A, Alireza A, Davood N, Mohammad B. A facile and efficient one-pot electrochemical synthesis of thiazole derivatives in aqueous solution. Chem Eur J 2015;20:1-6.

11. Charlotte A, Thomas BD, Elizabeth RJ. Silver-catalyzed enantioselective propargylation reactions of $n$-sulfonylketimines. Org Lett 2015;17:5340-3

12. Ahmed WN, Atheer MJ. Synthesis of some new sulfonamide derivatives based on 1,3,4-oxadiazole. J Chem Pharm Res 2015;7:300-6.

13. Ahmed WN, Ali RM. Synthesis of some new hetero cyclic compounds derived from O-(N-alkyl Sulfamide) benzohydrazide. Int J Basic Appl Sci 2016;4:99-109.

14. Ahmed WN, Aseel F. Synthesis of some new N-saccharin derivatives of possible biological activity. J Chem Pharm Res 2014;6:872-9.

15. Kany AA, Ahmed WN, Muthanna SF. Synthesis of new triazole and aza- $\beta$-lactam compounds derived from o-(N-propargyl)- sulfonamido benzoic acid of possible biological activity. Pharm Chem 2018;10:149-53.

16. Ahmed JQ, Ahmed WN. Synthesis, characterization, and antiinflammatory evaluation of some new 2-(3-Fluorobiphenyl-4-yl) propanoic acid derivatives. Int J Pharm Sci Res 2017;8:1598-605.

17. Ahmed TS, Ph.D. Thesis College of Science. Al-Nahrain University. 2008.

18. Ahmed WN. Synthesis of new 9H-Carbazole derivatives. Iraqi Natl J Chem 2013;199:50.

19. Furniss B, Hannaford AH, Smith P, Tatchell A. Vogel's Textbook of Practical Organic Chemistry. $5^{\text {th }}$ ed. London: Addison Wesley Longman; 1998. p. 1077.

20. Mal A, Ahmed SH. Synthesis and antimicrobial screening of new 9, 10-dihydro anthracene-9,10-endo- $\alpha, \beta$-succinimides bearing pharmacologically active components. Iraqi J Biotechnol 2017;16:40-52.

21. SirassuN T, Ranjith K, Nukala SK, Shaik YN, Vasudeva R. Synthesis and antibacterial activity of (1-aryl-1, 2, 3-triazole-4-yl) methyl esters of morpholine-3-carboxylic acid. Med Chem Res 2014;23:5321-7. 